\title{
A Numerical Study of Several Viscoelastic Fluid Models
}

\author{
Corina Putinar
}

January 13, 2016

\begin{abstract}
Viscoelastic fluids are a type of fluid with a solvent and immersed elastic filaments which create additional stresses on the fluid. The Oldroyd-B equations are a well accepted model of the flow of viscoelastic fluids but in extensional flows, a characteristic of flows where liquids approach or separate from each other, as the Wiessenberg number $(W i)$, a number that measures the relaxation time of the fluid, approaches infinity the stress of the polymer also goes to infinity. For small $W i$, the polymer stress remains bounded but as $W i$ gets bigger the polymer stress approaches a cusp shape until the solution eventually becomes unbounded. Modifications to the Oldroyd-B model have been proposed that keep the solutions bounded, such as the Polymer Diffusion, Giesekus Model, and Phan-Thien and Tanner model. Here we study how well these modifications approximate the Oldroyd-B model when the stress is very large. An ideal model for numerical simulations would be close to the Oldroyd-B model outside of a small region near the cusp or singularity but still be well-resolved near the singularity. Analysis has been done to see how the proposed solutions differ in regards to stress, time and other factors. When finding such results it is desirable to use minimal computing resources when resolving these near singular solutions. Several different modifications to the Oldroyd-B system with stress diffusion are investigated using MATLAB and discussed to identify which modifications perform the best in this flow geometry.
\end{abstract}




\section{Introduction}

Complex fluids display a mixture of viscous and elastic behaviors. These fluids are found in many industrial and natural settings. For example, bodily fluids, such as blood and mucus, and household products, such as shampoo and paint, under stress, can display complicated phenomena such as shear thinning and normal stress differences. One important flow geometry that is crucial to study is flow at extensional stagnation points. This flow situation arises in many common flow settings such as flow around a cylinder. At a stagnation point the velocity of the flow approaches zero, but large stresses develop as the immersed polymer coils are stretched. Using computer simulations to model this flow situation is complicated due to underlying nearsingularities in the flow for the Oldroyd-B model, one of the simplest closed continuum models of viscoelastic fluids.

Various modifications to the Oldroyd-B model have been proposed including the PTT model [7] and the Giesekus model [2]. We compare these models to the Oldroyd-B model as well as to a simple way of providing smoothing, namely adding polymer stress diffusion $[6,8]$. These three models are compared to the Oldroyd-B model in the extensional flow geometry.

A pseudo-spectral method will be used to compute the solutions and smoothness of the three models' solutions will be compared to the OldroydB solution. In Sec. 1.1 we introduce the Oldroyd-B model and in Sec. 1.2 we explain what is known about the Oldroyd-B model at extensional points and demonstrate the need for changes to the model. In Sec. 2 we describe the numerical method used in the simulations. Finally in Sec. 5 we introduce the other models and demonstrate our results for these modifications.

\section{$1.1 \quad$ Oldroyd-B Model}

As mentioned before, the Oldroyd-B model describes the flow of a viscoelastic fluid. For our simulations we are interested in an extensional background flow and hence we write this model in non-dimensional form as:

$$
\begin{array}{r}
\partial_{t} \mathbf{S}+\mathbf{u} \cdot \nabla \mathbf{S}-\left(\nabla \mathbf{u} \cdot \mathbf{S}+\mathbf{S} \cdot \nabla \mathbf{u}^{\top}\right)=\frac{-1}{W i}(\mathbf{S}-\mathrm{I}) \\
-\nabla p+\triangle \mathbf{u}=\beta \nabla \cdot \mathbf{S}+\mathbf{f} \\
\nabla \cdot \mathbf{u}=0 \\
\mathbf{f}=\left(\begin{array}{c}
-\sin (x) \cos (y) \\
\cos (x) \sin (y)
\end{array}\right),
\end{array}
$$


where $\mathbf{S}$ is the polymer stress tensor, $t$ is time, and $\mathbf{u}$ is the velocity of the fluid. The background force $\mathbf{f}$ prescribes a 4-roll mill type geometry which for $W i=0$ has the solution $\mathbf{u}=-\frac{1}{2} f$. The Wiessenberg number, $W i=\frac{\tau_{r}}{\tau_{f}}$, where $\tau_{r}$ is the relaxation time of the polymer and $\tau_{f}$ is the time scale flow of the fluid. The other non-dimensional parameters, $\beta$, is related to the fluid viscosity. In particular, $\beta \cdot W i$ is the ratio of polymer to solvent viscosity, which we fix $\beta \cdot W i=0.5$ in the following simulations $[1,5]$.

The Oldroyd-B equations and modifications are posed in a two dimensional periodic domain where $(x, y) \in[0,2 \pi)^{2}, \mathbf{u}(x, y)=\left(u_{1}, u_{2}\right)$ is the velocity and $\mathbf{S}=\left(\begin{array}{cc}S_{11} & S_{12} \\ S_{12} & S_{22}\end{array}\right)$ is the symmetric polymer stress tensor.

The Oldroyd-B system is designed to model the flow of a dilute polymer solution, where polymers are immersed in a Newtonian solvent. In the derivation of the Oldroyd-B model [1] a linear Hooke's law is assumed for the force due to extensions of polymer coils. To obtain a macroscopic equation for the stress tensor these polymers are averaged over all possible configurations. Hooke's Law, given by

$$
F=k X,
$$

where $F$ is the force, $k$ is a spring constant factor relating to the spring stiffness, and $X$ is distance, does not penalize infinite extension and this is one of the difficulties with the model [5].

\subsection{Behavior at extensional points}

It is simplest to see the behavior of the Oldroyd-B model at extensional points by assuming a linear background flow $\mathbf{u}=\alpha(x,-y)$ [9]. Substituting this in to Eq. (1) we see that the system decouples and one obtains a variable coefficient, linear partial differential equation for each component of the stress tensor. If we focus on $S_{11}$ we get the following PDE:

$$
\partial_{t} S_{11}+\alpha x \partial_{x} S_{11}-\alpha y \partial_{y} S_{11}-2 \alpha S_{11}=\frac{-1}{W i}\left(S_{11}-1\right) .
$$

Using the methods of characteristics there is a solution of form:

$$
S_{11}(x, y, t)=\frac{1}{1-2 \varepsilon}+e^{(2 \varepsilon-1) t} H\left(x e^{-\varepsilon t}, y e^{\varepsilon t}\right),
$$

where $\varepsilon=\alpha W i$ [4], $S_{11}$. In general the function $H$ must be determined by boundary conditions, however in simulations at extensional points we see 
that a unique behavior of the solution is along the direction of compression, here in the $y$ direction. Hence, we analyze the behavior of $H$ in $y$.

A solution of the form

$$
S_{11}=\frac{1}{1-2 \varepsilon}+A|y|^{\frac{1-2 \varepsilon}{\varepsilon}},
$$

matched the simulations very well. This solution can be obtained from Eq. (7) in the limit as $t \rightarrow \infty[9]$.

From Eq. 8 we can get three different types of solutions for the stress depending on the value of $\varepsilon$.

1. For $\frac{1-2 \varepsilon}{\varepsilon}>1$ the stress will remain smooth and bounded.

2. For $0<\frac{1-2 \varepsilon}{\varepsilon}<1$ the stress will approach a cusp (the rate of approach is unknown, though appears to be exponential in time).

3. For $\frac{1-2 \varepsilon}{\varepsilon}<0$ the stress will become divergent in time.

Substituting $\varepsilon=\alpha W i$, we have the approximate regimes for $W i$,

1. $0<W i<0.5$ : The solution is at least differentiable.

2. $0.5<W i<1$ : The solution approaches a (bounded) cusp (not differentiable).

3. Wi $>1$ : The solution diverges in time (unbounded, not differentiable).

[9] These three regimes will be demonstrated in Sec. 3.

\section{Spectral method}

A pseudo-spectral method is used to simulate the Oldroyd-B model, the Fast Fourier transform is taken of the Oldroyd-B equation allowing analysis of numerical accuracy. Both the spectral method and Oldroyd-B model have been calculated with $N$ at multiple values: 64, 128, 256, 512, and 1024 . Given the cost of numerical implementation and accuracy of solutions, $N$ at 512 is the most reasonable resolution to get accurate but not sparse data since it would take a large quantity of time and computational power to get enough data to analyze $N$ at 1024 .

MATLAB has been used with the following parameters to gather data and simulations of the Oldroyd-B system given in Eqs. (1)-(4). 
1. $N$

The variable $N$ describes the frequency of the data, at a larger $N$ there is more data resulting in higher accuracy. For our Oldroyd-B simulations, $N$ has been taken and analyzed at the following values:

\begin{tabular}{c|c}
$\mathrm{N}$ & Analysis of data \\
\hline 64 & Too sparse for analysis \\
\hline 128 & Too spare for analysis \\
\hline 256 & Good value for representing data \\
\hline 512 & Good value for representing data \\
\hline 1024 & Good value but computational cost is high
\end{tabular}

2. $W i$

The variable $W i$, as previously described, affects the divergence of the Oldroyd-B model. For our testing purposes, $W i$ has been used at 0.3 , 0.7 , and 3.0. Those values were chosen so that one can easily analyze the behavior at a range of values, since the behavior of $W i$ at 0.7 mimics that of $W i$ at 1.0. As we will see in the graphs later on, the $W i$ value at 3.0 has data that tends towards $\infty$ much faster than at the values of 0.3 or at 0.7 . As seen through our graphs, the data when $W i$ is equal to 0.3 does not numerically reach a high enough point on the y-axis to get a fully accurate pattern of results. Therefore, we have found that at $W i$ equal to 0.7 our results are able to be retrieved without a steep increase to $\infty$. These values were taken at time 5 and 7 so that enough time has elapsed to properly estimate the behavior of $W i$.

\section{Simulations of Oldroyd-B behavior at ex- tensional points}

We show results from numerical simulations of the Oldroyd-B system given in Eqs. (1)-(4) using MATLAB and the numerical algorithm outlined in Sec. 2. In each figure we graph $S_{11}(\pi, y, t)$ to demonstrate the different behavior predicted by the analysis of the PDE from Eq. (6).

For $W i=0.3$ we should have a smooth bounded solution, and in Fig. 1 (a) we display the results with $N=256$ at $t=5$. For $W i=0.7$ we should have a cusp solution, and in Fig. 1 (b) we display the results with $N=256$, at $t=5$, which appears to be approaching a bounded, cusp. Finally, for 
$W i=3.0$ we should have a diverging solution, and in Fig. 1 (c) we display the results with $N=256$, at $t=5$, and this solution appears to be diverging in time. For other $W i$ values we have a solution that is not a clear bound, cusp, or divergent solution. For example, Wi at 0.5 has a solution that is closer to a cusp shape but still has bounded properties.

As we can see, the stress, $S_{11}(\pi, y, t)$, is dependent on $W i$ because it changes rapidly and diverges when $W i$ is increasing.
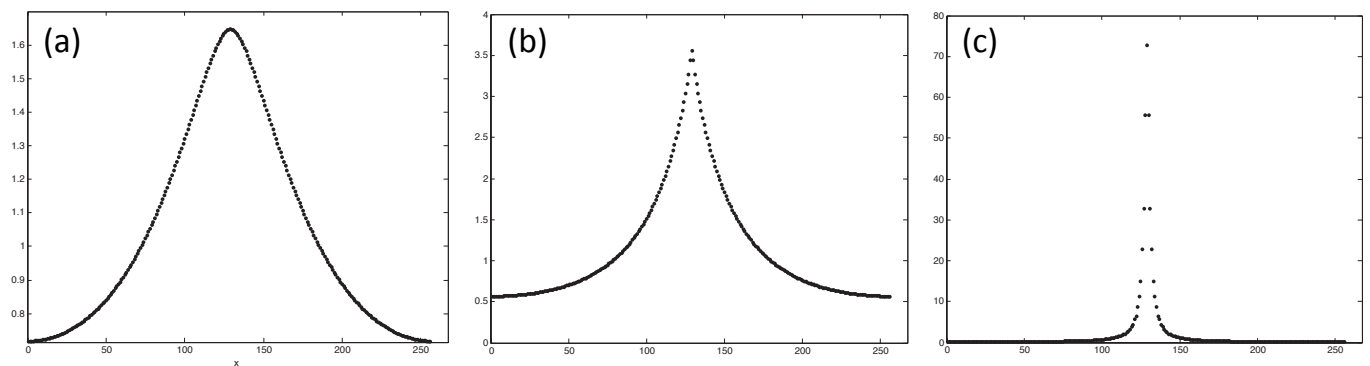

Figure 1: Plots of $S_{11}(\pi, y, t)$ for (a) $W i=0.3$ (b) $W i=0.7$ (c) $W i=3.0$. This shows the frequency, $k$ against the Fast Fourier Transform of $S$. The range for $k$ is different for each image so that one can see how the density increases as $N$ increases.

\section{Spectra of Stress}

Taking the Fourier Transform of the Oldroyd-B model gives us the amount of frequency that stress, $S$. With graphs (a), (b), and (c) of Figure 2, a graph of $\hat{S}$, the Fourier Transform of $\mathrm{S}$, has been configured. $\hat{S}$ can show us how the frequency, $N$, impacts the Oldroyd-B model.

Figure 3 shows that as $N$, a number denoting the gridpoints, decreases the graph becomes much more sparse, and thus approximated. Given these parameters, $N$ at 64 would be too sparse for our model but $N$ at 256 is a better idea of what needs to be analyzed. 

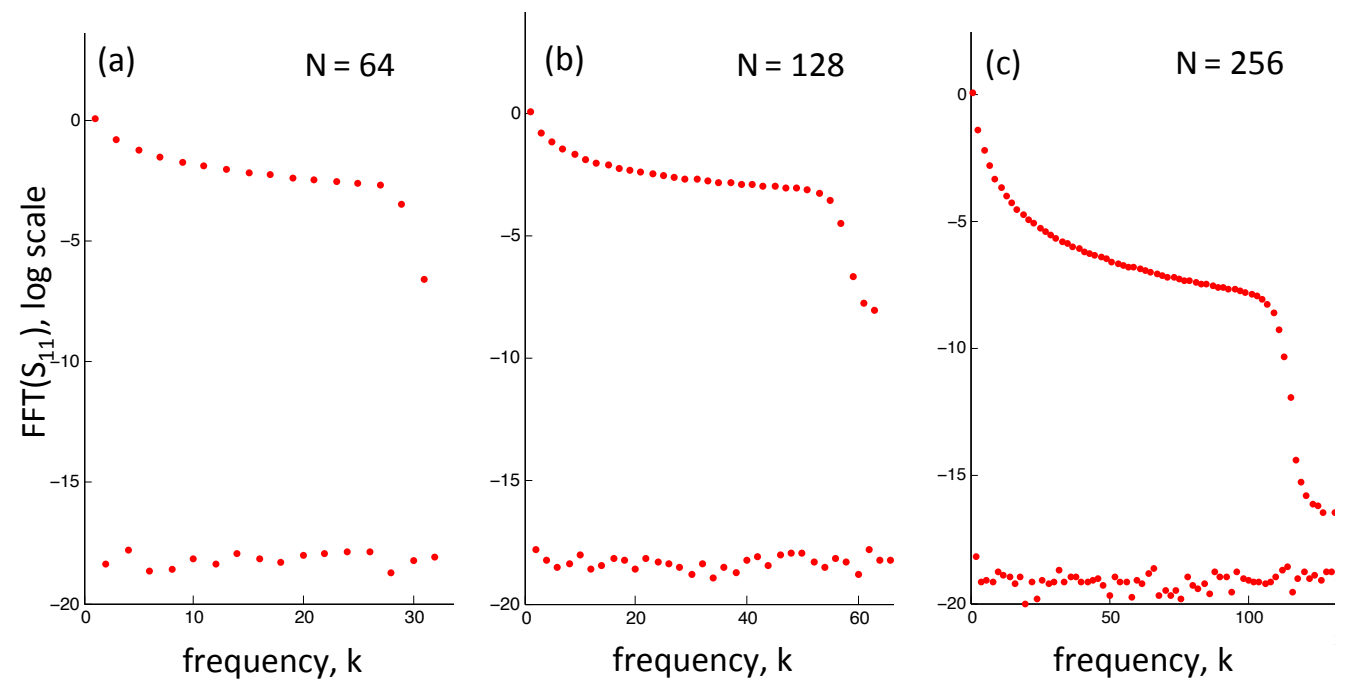

Figure 2: Plots of $\widehat{S_{11}}(\pi, k, t)$ for (a) $N=64$ (b) $N=128$ (c) $N=256$.

\section{Modifications}

\subsection{New Equations}

Recall, the Oldroyd-B equation:

$$
\partial_{t} S+\mathbf{u} \cdot \nabla S-\left(\nabla u \cdot S+S \cdot \nabla u^{\top}\right)=\frac{-1}{W i}(S-\mathrm{I})
$$

The Oldroyd-B equation can be sub-divided into multiple equations.

We can set the right hand side of the equation equal to a variable, $R(S)$ :

$$
R(S)=\frac{-1}{W i}(S-I)
$$

1. Polymer Diffusion The Polymer Diffusion is derived from equation (9) by adding the wave vector and a delta of stress.

$$
R(S)=\frac{-1}{w i}(S-I)+k \triangle S
$$

2. PTT

The Phan-Thien and Tanner model comes from transient model network theory and assumes models can break and reform. [3]. 


$$
R(S)=\frac{-1}{w i}(S-I)-k S \operatorname{tr}(S)
$$

3. Giesekus

The Giesekus model is derived using a simple dumbbell model by replacing equation (9) with:

$$
R(S)=\frac{-1}{w i}(S-I)-k(S)^{2}
$$

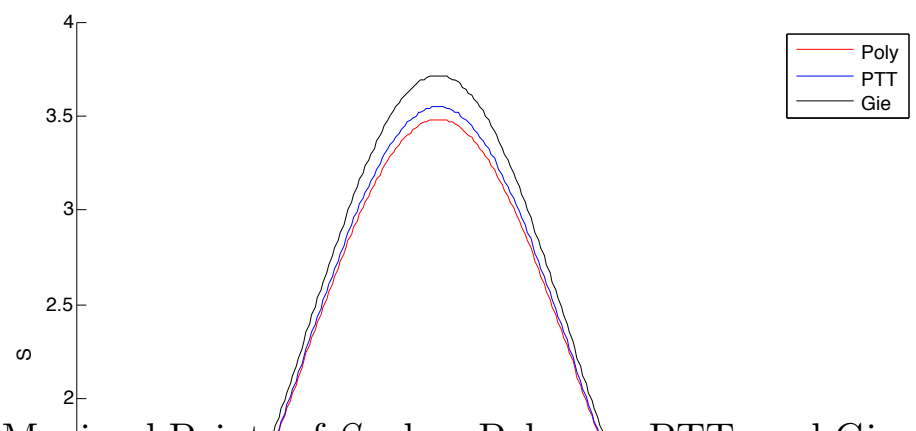

Figure 3: Maximal Point of $S$ when Polymer, PTT, and Giesekus Diffusions are used withs $-W i=0.70$ and $N=256$.

This imâge is zoomed to best display the maximal points of each graph. This image uses the parameters of Wi at 0.70 and $\mathrm{N}$ at 256. It shows the

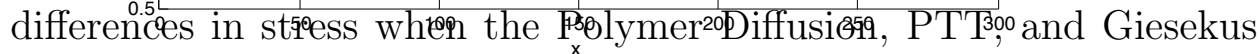
equations are used.

\section{$5.2 \quad$ Numerical Results}

These following simulations are the 'best' approximation to the Oldroyd-B solution with 'minimal' computational resources and 'maximal' agreement. We have compared three possible regularizations of the Oldroyd-B Model, as mentioned in Section 5.1, The Polymer Diffusiofrd, Models, at the same level of $k=0.001$. With these comparisons, there are two classes of solution types examined. 1) A cusp solution which is when $W i$ is between 0.5 and 1 . As we can see from Section 3 , the cusp is approached exponentially in time. By analyzing our results, we can see that as $t$ increases, 
the cusp is displaying properties that it would when $t=\infty$. Since we want to minimize computational resources, the time $t=5$ is enough to show the cusp figure. 2) The divergent solution which occurs as $W i$ increases, to use 'minimal' computational resources we have found a $W i=3.0$ simulation to be a good approximation of the divergent pattern. The following simulations have been completed with $W i=0.7$ and $W i=3.0$ at time $t=5$ and $t=7$ with $N=256$ and $N=1024$. 


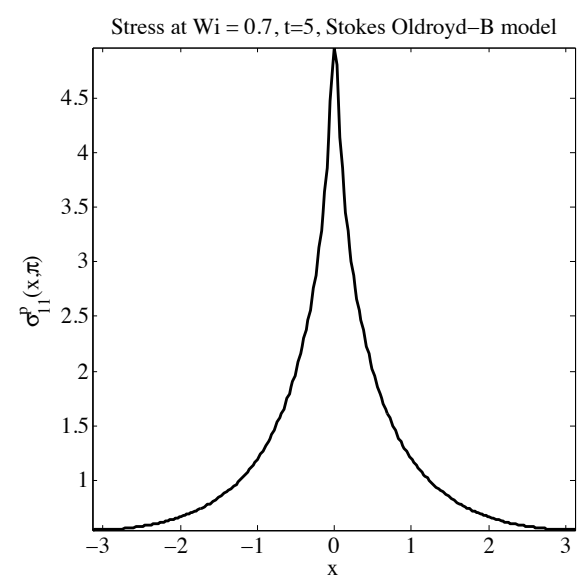

(a) Stress, $\mathrm{Wi}=0.7, \mathrm{t}=5, \mathrm{~N}=256$

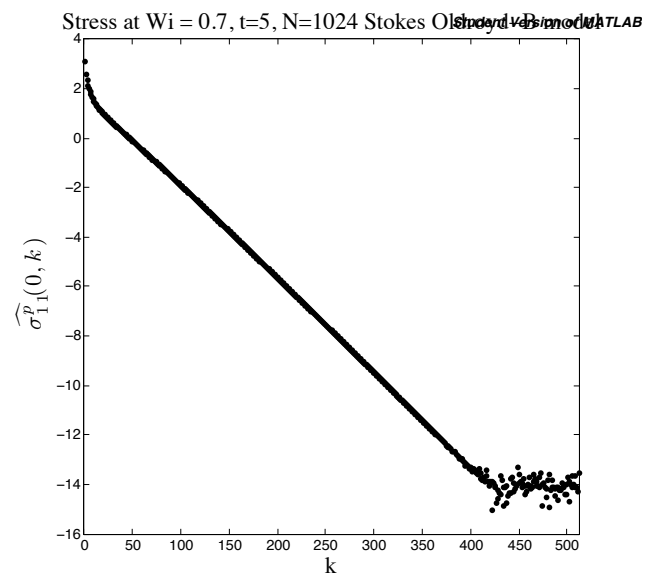

(a) Stress, $\mathrm{Wi}=0.7, \mathrm{t}=5, \mathrm{~N}=1024$

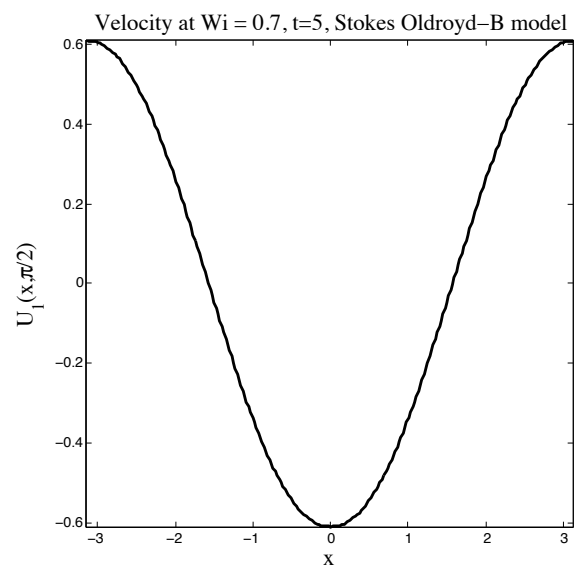

(b) Velocity, $\mathrm{Wi}=0.7, \mathrm{t}=5, \mathrm{~N}=256$

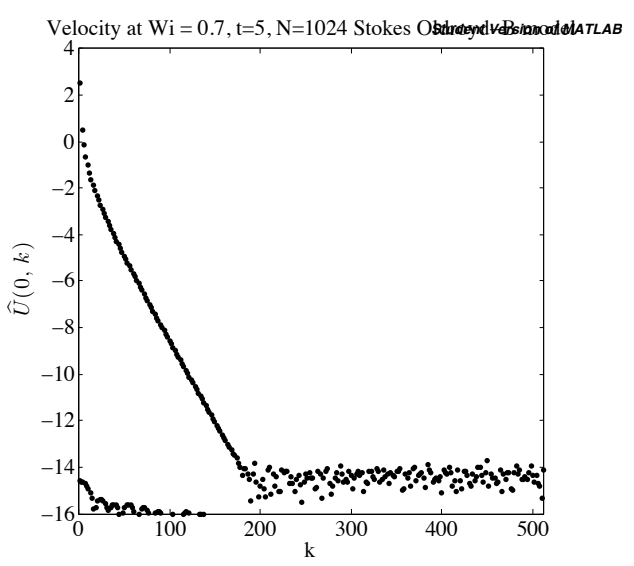

(b) Velocity, $\mathrm{Wi}=0.7, \mathrm{t}=5, \mathrm{~N}=1024$

In Figure 4a we can see that since the cusp grows exponentially in time, we were able to find a time that portrayed the 'cusp' like shapesudatitshidanarthe

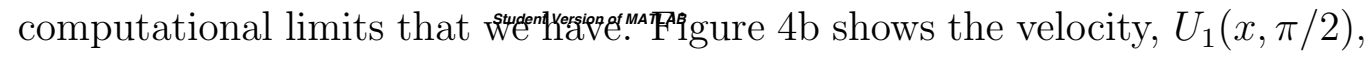
following the same axes as Figure 4a. The images 5a and 5b show how our simulation of the Stokes-Oldroyd-B model $(N=1024)$ is well resolved at a degree of $10^{-14}$. 


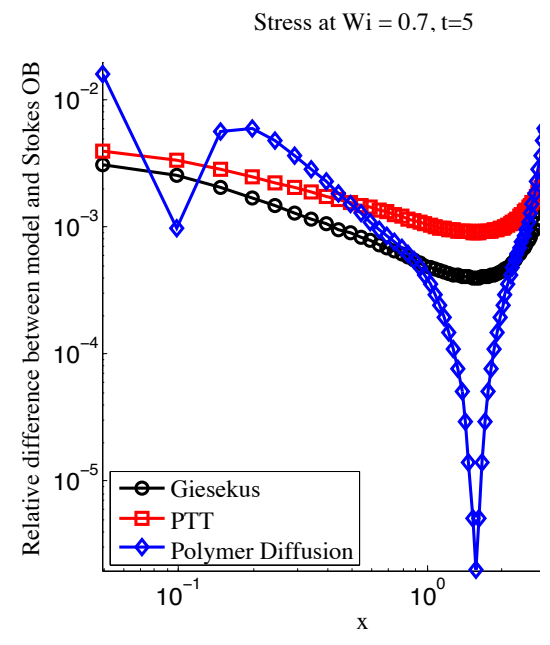

(a) Stress, $\mathrm{Wi}=0.7, \mathrm{t}=5, \mathrm{~N}=256$

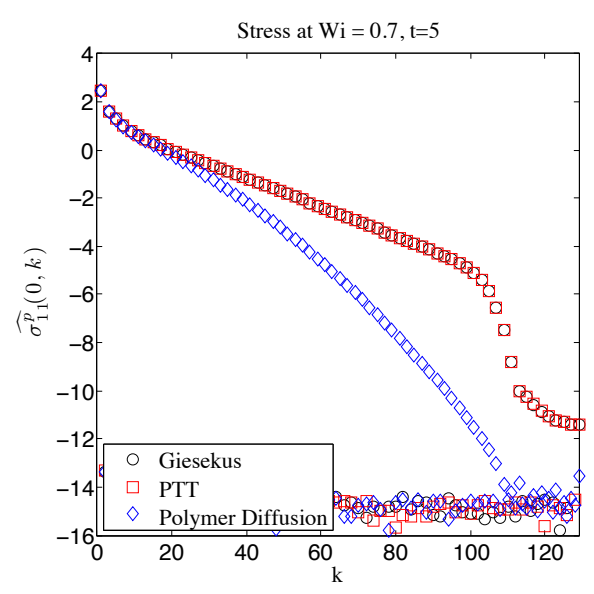

(a) Stress, $\mathrm{Wi}=0.7, \mathrm{t}=5, \mathrm{~N}=256$

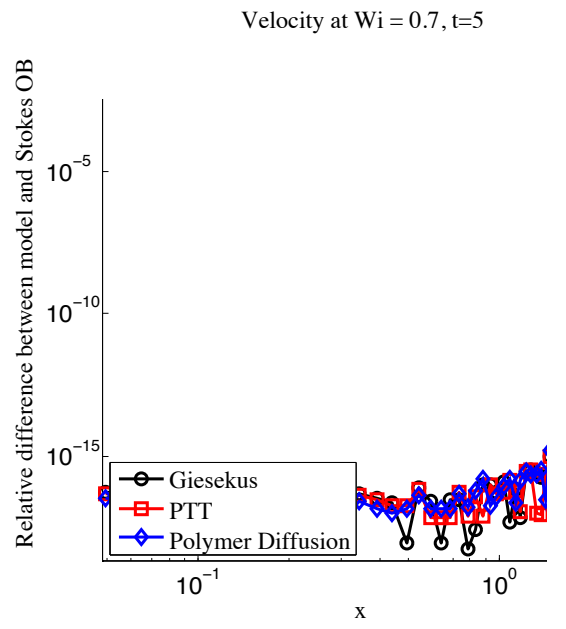

(b) Velocity, $\mathrm{Wi}=0.7, \mathrm{t}=5, \mathrm{~N}=256$

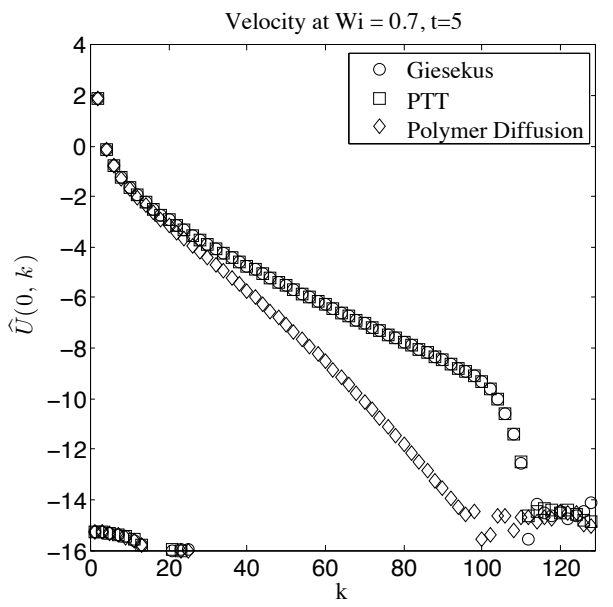

(b) Velocity, $\mathrm{Wi}=0.7, \mathrm{t}=5, \mathrm{~N}=256$

In Figure 6a we plot the relative difference of one component of the poly-

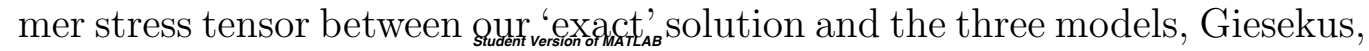

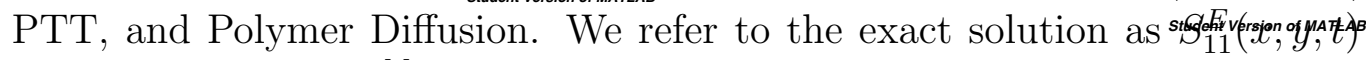
and the model as $S_{11}^{M}(x, y, t)$. The most singular behavior of our solution is along the line of compression, i.e. $(0, y)$ for $-\pi \leq y \leq \pi$, but due to 
symmetries in the problem we plot here

Relative difference $=\frac{\left|S_{11}^{E}(0, y, t)-S_{11}^{M}(0, y, t)\right|}{\left|S_{11}^{E}(0, y, 5)\right|}$, for $0 \leq y \leq \pi / 2$ and $\mathrm{t}=5$.

The time $t=5$ is chosen to compare with the 'exact' solution which was also at $t=5$. We notice that all three models capture the exact behavior of the Stokes-Oldroyd-B solution to within $\sim 2-3$ digits of accuracy. Note that the $x$-axis is plotted on a logarithmic scale so the details of the solution near the extensional point $(x, y)=(0,0)$ are displayed. We see that the polymer diffusion model is the least accurate at the extensional point but away from the extensional point all the models are quite similar. Figure $6 \mathrm{~b}$ shows the relative difference between the 'exact' solution and the 3 models for the velocity along the line $(x, y)=(\pi / 4, y)$ for $0 \leq y \leq \pi / 2$.

Figure 7a shows the Fourier transform of the $(1,1)$ component of the polymer stress along the line of compression for each of the 3 models, $\widehat{S}_{11}^{M}(0, k)$, where $k$ is the wave vector. We see that using $N=256$ gives a solution which is resolved to machine accuracy for the polymer diffusion model while both the Giesekus and PTT model show decay in the wave number up to $10^{-6}$ at this time $(t=5)$. Results are similar for the Fourier transform of the velocity, $\hat{u}^{M}(0, k)$, plotted in Fig. $7 \mathrm{~b}$ but as the velocity is one order smoother than the stress there is more decay in the modes for the Giesekus and PTT models. 


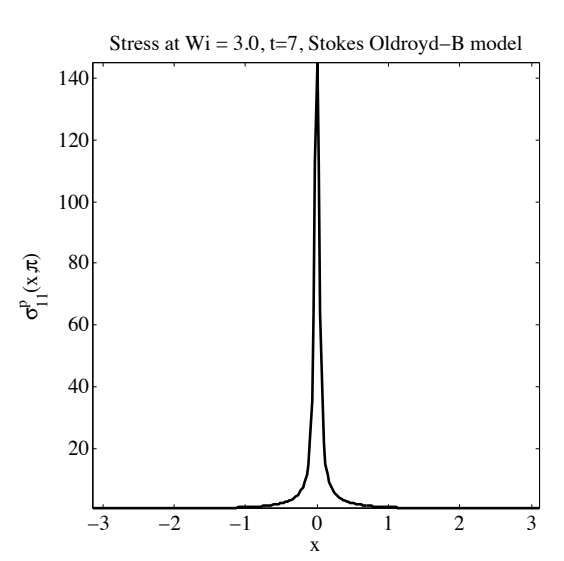

(a) Stress, $\mathrm{Wi}=3.0, \mathrm{t}=7, \mathrm{~N}=1024$

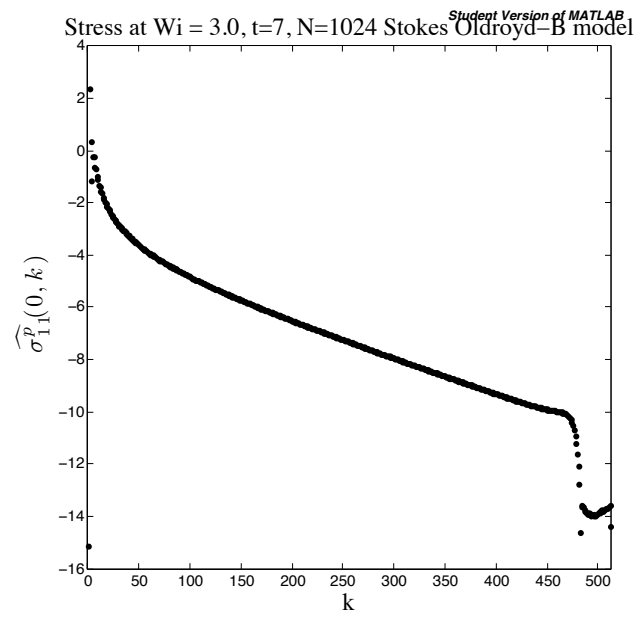

(a) Stress, $\mathrm{Wi}=3.0, \mathrm{t}=7, \mathrm{~N}=1024$

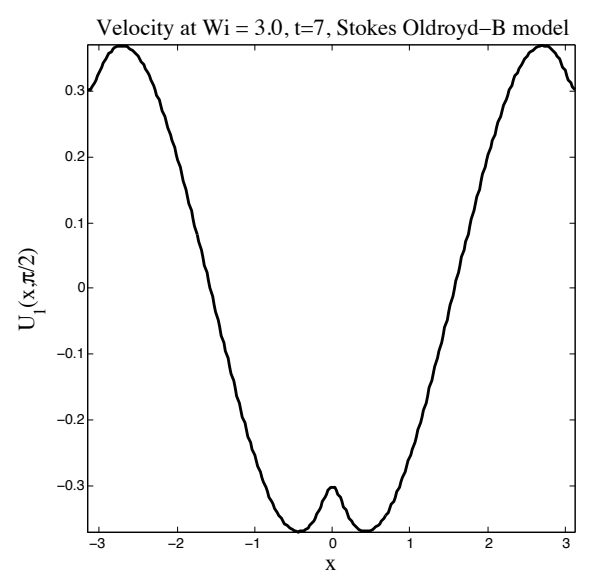

(b) Velocity, $\mathrm{Wi}=3.0, \mathrm{t}=7, \mathrm{~N}=1024$

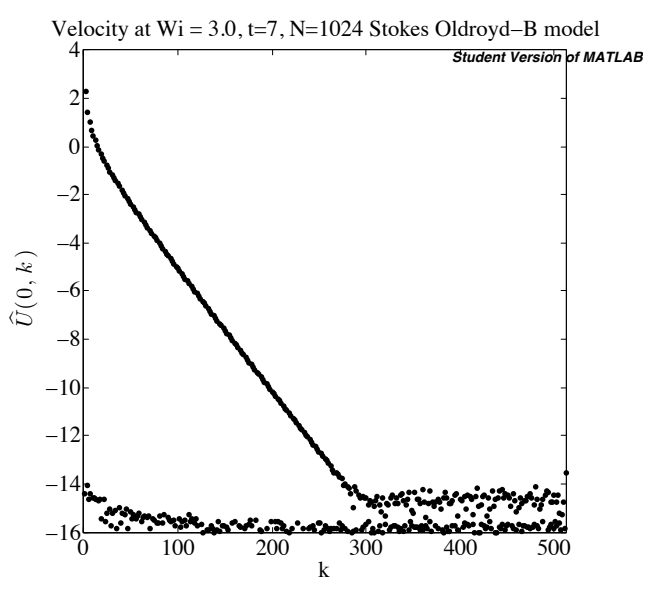

(b) Velocity, $\mathrm{Wi}=3.0, \mathrm{t}=7, \mathrm{~N}=1024$

In figure $8 \mathrm{a}$ we plot the 'divergent' model, as stated in the Section 3

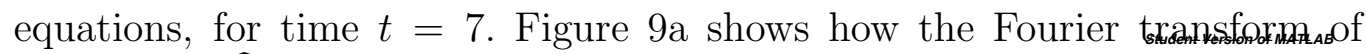

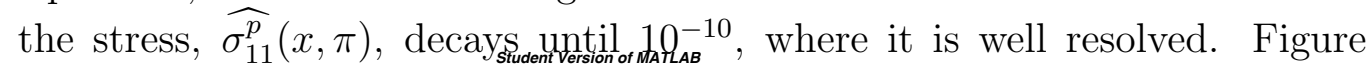
11a shows the Fourier transform of the velocity, $\widehat{U_{1}}(x, \pi / 2)$, and how it is a well-resolved simulation of Stokes-Oldroyd-B model $(N=1024)$. 
Stress at $\mathrm{Wi}=3.0, \mathrm{t}=7$

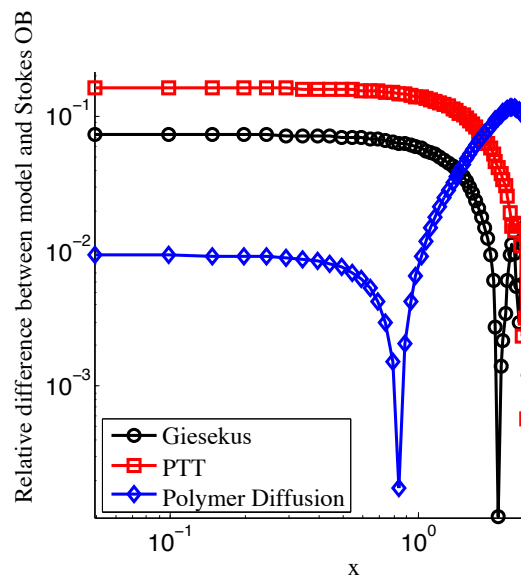

(a) Stress, $\mathrm{Wi}=3.0, \mathrm{t}=7, \mathrm{~N}=256$

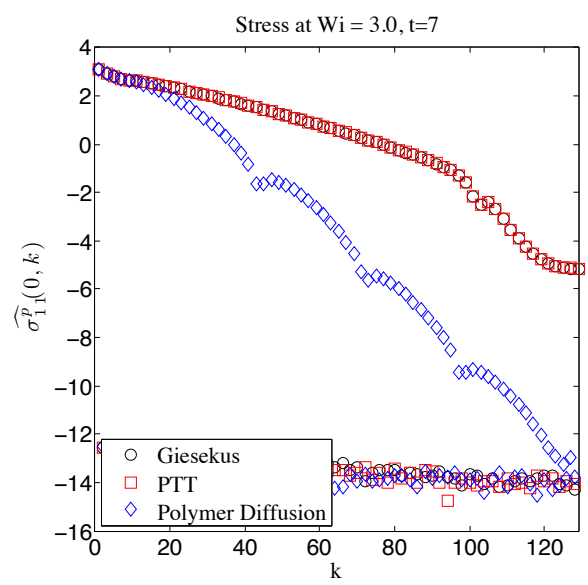

(a) Stress, $\mathrm{Wi}=3.0, \mathrm{t}=7, \mathrm{~N}=256$

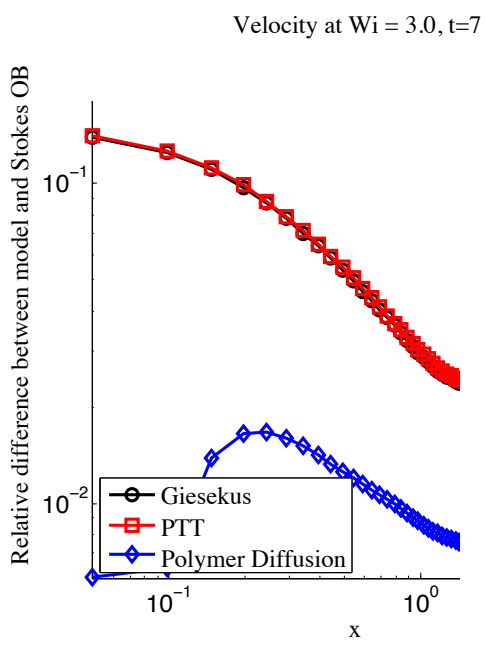

(b) Velocity, $\mathrm{Wi}=3.0, \mathrm{t}=7, \mathrm{~N}=256$

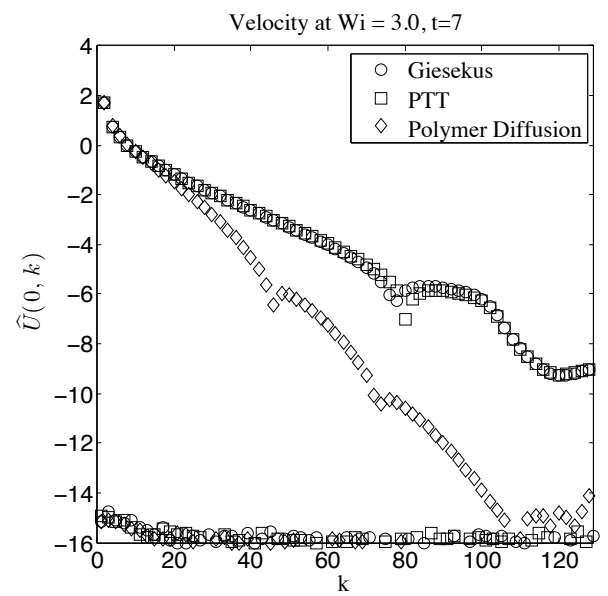

(b) Velocity, Wi=3.0, t=7, N=256

These figures, like Figures 6a-7b, plot the relative difference between our

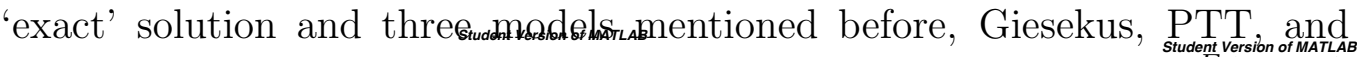
Polymer Diffusion. Once again, we refer to the exact solution as $\boldsymbol{\tau}_{11}^{E}(x, y, t)$ and the model as $\boldsymbol{\tau}_{11}^{M}(x, y, t)$. In this case, our relative difference is taken at $\mathrm{t}$ $=7$ for $0 \leq y \leq \frac{\pi}{2}$. The time $t=7$ is chosen here to compare with the 'exact' 
solution since $W i$ was increased to 3.0. We can see that the new equations capture the Stokes-Oldroyd-B solution between 1-2 digits of accuracy. The polymer diffusion model is least accurate at the extensional point but regains accuracy away from the extensional point. Figure 10b shows the relative difference between the 'exact' solution and the three models along the line $(x, y)=(\pi / 4, y)$ for $0 \leq y \leq \pi / 2$. Figure 11a shows the Fourier transform of the $(1,1)$ component of the polymer stress along the line of compression for each of the 3 models, $\widehat{\tau}_{11}^{M}(0, k)$, where $k$ is the wave vector. We see that the PTT and Giesekus models show decay in the wave number for up to $\left(10^{-} 2\right)$, which is less than $\left(10^{-} 6\right)$ which we saw for $6 \mathrm{~b}$. The polymer diffusion model is still resolved to machine accuracy. Figure 11b shows the relative difference between the 'exact' solution and the three models along the line $(x, y)=(\pi / 4, y)$ for $0 \leq y \leq \pi / 2$. 


\section{Conclusion}

We saw that the Stokes-Oldroyd-B model has exponential-in-time singularities which occur in extensional flows. For the values of Wiessenberg at $W i=0.3, W i=0.7$, and $W i=3.0$, we've noticed that the Stokes-Oldroyd$\mathrm{B}$ will diverge, and lose a cusp-like shape and instead imitate a line shape, if $W i$ is $\approx 3, \frac{1-2 \alpha W i}{\alpha W i}<0$, will imitate a cusp if $W i$ is $\approx 0.7,0<\frac{1-2 \alpha W i}{\alpha W i}<1$, and will have a smooth and bounded result if $W i$ is $\approx 0.3, \frac{1-2 \alpha W i}{\alpha W i}>1$. Our goal was to compare the three modifications as described in 5.1, Polymer Diffusion, PTT, and Giesekus, to the Stokes Oldroyd-B model to see if they could match the behavior of the SOB model at lower resolutions. Through our simulations, we found that all three modifications do a good job approaching the Stokes-Oldroyd-B model in the short term, but at higher values the PTT and Giesekus models suffer from the same problems as the Stokes-Oldroyd-B model for longer times, such as a blow-up of data at a low resolution. The Polymer Diffusion model, unlike the other models, can be simulated at long times while keeping accuracy. This can also bee seen in the figures $6 \mathrm{a}, 7 \mathrm{a}$, and 11a.

Our study on Stokes-Oldroyd-B models and variations had limitations on the computational time and resources we could use. Therefore, we had to derive an analysis from lower resolutions which led to approximative results rather than more refined results at a higher $W i$ and $N$.

\section{Acknowledgements}

I would like to thank Professor Becca Thomases for her continued support and guidance throughout this project.

\section{References}

[1] R Byron Bird, Robert C Armstrong, and Ole Hassager. Dynamics of polymeric liquids. vol. 1: Fluid mechanics. 1987.

[2] H Giesekus. A simple constitutive equation for polymer fluids based on the concept of deformation-dependent tensorial mobility. Journal of Non-Newtonian Fluid Mechanics, 11(1):69-109, 1982. 
[3] Robert Guy and Becca Thomases. Computational challenges for simulating strongly elastic flows in biology.

[4] Fritz John. Partial differential equations, 1982.

[5] Ronald G Larson. The Structure and Rheology of Complex Fluids, volume 4. Oxford university press New York, 1999.

[6] R Sureshkumar and Antony N Beris. Effect of artificial stress diffusivity on the stability of numerical calculations and the flow dynamics of timedependent viscoelastic flows. Journal of Non-Newtonian Fluid Mechanics, 60(1):53-80, 1995.

[7] Nhan Phan Thien and Roger I Tanner. A new constitutive equation derived from network theory. Journal of Non-Newtonian Fluid Mechanics, 2(4):353-365, 1977.

[8] Becca Thomases. An analysis of the effect of stress diffusion on the dynamics of creeping viscoelastic flow. Journal of Non-Newtonian Fluid Mechanics, 166(21):1221-1228, 2011.

[9] Becca Thomases and Michael Shelley. Emergence of singular structures in oldroyd-b fluids. Physics of Fluids (1994-present), 19(10):103-103, 2007. 